\title{
ON STIELTJES POLYNOMIALS AND GAUSS-KRONROD QUADRATURE
}

\author{
FRANZ PEHERSTORFER
}

\begin{abstract}
Let $D$ be a real function such that $D(z)$ is analytic and $D(z) \neq 0$ for $|z| \leq 1$. Furthermore, put $W(x)=\sqrt{1-x^{2}}\left|D\left(e^{i \varphi}\right)\right|^{2}, x=\cos \varphi$, $\varphi \in[0, \pi]$, and denote by $p_{n}(\cdot, W)$ the polynomial which is orthogonal on $[-1,+1]$ to $\mathbb{P}_{n-1}\left(\mathbb{P}_{n-1}\right.$ denotes the set of polynomials of degree at most $n-1)$ with respect to $W$. In this paper it is shown that for each sufficiently large $n$ the polynomial $E_{n+1}(\cdot, W)$ (called the Stieltjes polynomial) of degree $n+1$ which is orthogonal on $[-1,+1]$ to $\mathbb{P}_{n}$ with respect to the sign-changing function $p_{n}(\cdot, W) W$ has $n+1$ simple zeros in $(-1,1)$ and that the interpolation quadrature formula (called the Gauss-Kronrod quadrature formula) based on nodes which are the $2 n+1$ zeros of $E_{n+1}(\cdot, W) p_{n}(\cdot, W)$ has all weights positive.
\end{abstract}

\section{INTRODUCTION}

In the following we say that a function $w$ is a weight function on $[-1,1]$ if $w \in L^{2}[-1,1]$ and $w(x) \geq 0$ for $x \in(-1,1)$. In 1894, in one of his letters to Hermite, Stieltjes introduced and characterized for the Legendre weight, i.e., the constant weight $w(x)=1$, a remarkable class of polynomials $E_{n+1}(x):=$ $E_{n+1}(x, w)=x^{n+1}+\cdots$ satisfying the following orthogonality conditions (with respect to a sign-changing function):

$$
\int_{-1}^{1} x^{j} E_{n+1}(x, w) P_{n}(x, w) w(x) d x=0 \text { for } j=0,1, \ldots, n,
$$

where $P_{n}(x, w)=x^{n}+\cdots$ denotes the monic polynomial of degree $n$ which is orthogonal to $\mathbb{P}_{n-1}\left(\mathbb{P}_{m}\right.$ denotes as usual the set of real polynomials of degree at most $m$ ) on $[-1,1]$ with respect to $w$. For the Legendre weight, Stieltjes made the conjecture that the zeros of $E_{n+1}$ are all in $(-1,1)$ and interlace with the zeros of the Legendre polynomial $P_{n}$. In 1934, G. Szegö [18] proved Stieltjes' conjecture. In addition he proved that the conjecture holds true for the Gegenbauer weight function $w(x, \lambda)=\left(1-x^{2}\right)^{\lambda-1 / 2}, 0<\lambda \leq 2$.

Received June 3, 1989.

1980 Mathematics Subject Classification (1985 Revision). Primary 65D32; Secondary 42C05.

Key words and phrases. Stieltjes polynomials, orthogonal polynomials, Gauss-Kronrod quadrature formulas. 
In 1964, Kronrod [8] considered quadrature formulas-now known under the name "Gauss-Kronrod quadrature formulas" - with the following properties:

$$
\int_{-1}^{1} f(x) w(x) d x=\sum_{\nu=1}^{n} \sigma_{\nu, n} f\left(x_{\nu, n}\right)+\sum_{\mu=1}^{n+1} \lambda_{\mu, n} f\left(y_{\mu, n}\right)+R_{n}(f),
$$

where $x_{\nu, n}$ are the Gaussian nodes for the weight function $w$, i.e., $x_{\nu, n}$ are the zeros of $P_{n}(\cdot, w)$, and the nodes $y_{\mu, n}$ and quadrature weights $\sigma_{\nu, n}, \lambda_{\mu, n}$ are chosen so as to maximize the degree of exactness of (1.2); thus $R_{n}(f)=0$ for all $f \in \mathbb{P}_{3 n+1}$ at least. It turned out (as it is not hard to see) that the exactness condition $R_{n}(f)=0$ for $f \in \mathbb{P}_{3 n+1}$ is equivalent to the fact that $E_{n+1}(x, w):=\prod_{\mu=1}^{n+1}\left(x-y_{\mu, n}\right)$ satisfies the orthogonality condition (1.1). Thus, the $n+1$ zeros of the Stieltjes polynomial $E_{n+1}(x, w)$ are the $n+1$ nodes $y_{\mu, n}$ required in the Gauss-Kronrod rule. Of foremost interest are weight functions for which the Gauss-Kronrod quadrature formula has the property that

(i) all $n+1$ nodes $y_{\mu, n}$ are in $(-1,1)$ and are simple (i.e., that all zeros of the Stieltjes polynomial $E_{n+1}(\cdot, w)$ are in $(-1,+1)$ and are simple).

Also desirable are weight functions which have in addition to (i) the properties

(ii) the nodes $y_{\mu, n}$ and $x_{\nu, n}$ separate each other (i.e., the $n+1$ zeros of $E_{n+1}(x, w)$ separate the $n$ zeros of the orthogonal polynomial $\left.P_{n}(x, w)\right)$; and

(iii) all quadrature weights are positive.

By a result of Monegato [10], property (ii) is equivalent to the positivity of the quadrature weights $\lambda_{\mu, n}, \mu=1, \ldots, n+1$, and thus property (iii) requires in fact the positivity of the $\sigma_{\nu, n}$ 's only. As already mentioned for the Gegenbauer weight function $w(x, \lambda)=\left(1-x^{2}\right)^{\lambda-1 / 2}, 0<\lambda \leq 2$, properties (i) and (ii) have been shown by Szegö [18], while property (iii) has been established for $0<\lambda \leq 1$ by Monegato [11]. For further (algebraic) investigations on Gauss-Kronrod quadrature with respect to Gegenbauer and Jacobi weight functions, see Gautschi and Notaris [2]. Quite recently, it has been shown independently by Notaris [13] and by the author [14] that weight functions of the form $w\left(x, s_{m}\right)=\left(1-x^{2}\right)^{1 / 2} / s_{m}(x)$, where $s_{m}$ is an arbitrary polynomial which is positive on $[-1,1]$, satisfy all three properties stated above for $n \geq m$. For the special case $s_{2}(x)=(1+\gamma)^{2}-4 \gamma x^{2}$, this was first discovered by Gautschi and Rivlin [3]. So far, only these two families of weight functions are known which satisfy (i)-(iii). In this paper we describe another large class of weight functions which have these three properties. More precisely, it will be shown that every weight function of the form

$$
W(x)=\sqrt{1-x^{2}}\left|D\left(e^{i \theta}\right)\right|^{2}, \quad x=\cos \theta, \theta \in[0, \pi],
$$

where $D(z)$ is analytic, $D(z) \neq 0$ for $|z| \leq 1$, and $D$ is real on $\mathbb{R}$, satisfies properties (i)-(iii) for sufficiently large $n$. As a very special case of this result, we obtain the above mentioned fact-recalling that every polynomial $s_{m}$ of degree $m$ which is positive on $[-1,+1]$ can be represented uniquely in the 
form $s_{m}(x)=\left|h_{m}\left(e^{i \theta}\right)\right|^{2}, x=\cos \theta, \theta \in[0, \pi]$, where $h_{m}$ is a real polynomial of degree $m$ which has all its zeros outside of the closed unit disk-that $w\left(x, s_{m}\right)=\sqrt{1-x^{2}} / s_{m}(x)$ satisfies the three properties for $n \geq n_{0}$. Note however, that we do not get the exact lower bound $n_{0}=m$ by our general approach. Naturally, the question arises whether the assumptions on the function $D$ can be weakened to $D(z)$ analytic and $D(z) \neq 0$ for $|z|<1$, and the existence of the Lebesgue integral of $W$. That this is not possible without imposing additional conditions on $D$ follows from a result of Rabinowitz [17] which says that Jacobi weight functions of the form

$$
(1-x)^{\alpha}(1+x)^{\beta}=2^{-(\alpha+\beta)}\left|\left(1-e^{i \theta}\right)^{\alpha}\left(1+e^{i \theta}\right)^{\beta}\right|^{2},
$$

$x=\cos \theta, \theta \in(0, \pi), \alpha=-1 / 2$, and $-1 / 2<\beta<1 / 2$, resp. $\alpha=-1 / 2$ and $1 / 2<\beta \leq 3 / 2$, have at least one Kronrod node outside of the interval $[-1,1]$, when $n$ is even, resp. when $n$ is odd. Other examples are weight functions of the form $(1-x)^{\alpha}(1+x)^{\beta} / s_{m}, \alpha, \beta \in\{-1 / 2,1 / 2\}$ and $\{\alpha\} \cap\{\beta\} \neq\{1 / 2\}, s_{m}$ a positive polynomial on $[-1,1]$ of degree $m$. As has been demonstrated by Notaris [13], such weight functions have the property that the smallest or largest Kronrod node is +1 or -1 , i.e., a boundary point of the support $[-1,1]$. Apart from this fact they satisfy properties (i)-(iii).

\section{Preliminary Results}

Notation. Let

$$
p_{n}(x):=p_{n}(x, w):=k_{n} \prod_{\nu=1}^{n}\left(x-x_{\nu, n}\right)
$$

denote the orthonormal polynomial of degree $n$, i.e.,

$$
\int_{-1}^{1} p_{n}(x, w) p_{m}(x, w) w(x) d x=\delta_{n, m} \text { for } n, m \in \mathbb{N}_{0} .
$$

Furthermore, the $n$th function of second kind with respect to the weight function $w$ is denoted and defined by

$$
\begin{aligned}
q_{n}(y) & :=q_{n}(y, w):=\int_{-1}^{1} \frac{p_{n}(x, w)}{y-x} w(x) d x \\
& =\sum_{j=n}^{\infty}\left(\int_{-1}^{1} x^{j} p_{n}(x, w) w(x) d x\right) y^{-(j+1)} \text { for } y \in \mathbb{C} \backslash[-1,1]
\end{aligned}
$$

where the last expansion is convergent for $|y|>1$.

First we give a simple proof of a slightly extended version of Stieltjes' result about the orthogonality of his polynomials (see [12, p. 138]).

Lemma 1 (Stieltjes). Let $w$ be a weight function on $[-1,+1]$, and let $E_{n+1}(x)$ $=x^{n+1}+\cdots$ be a polynomial of degree $n+1$. Then

$$
\int_{-1}^{1} x^{j} E_{n+1}(x) p_{n}(x, w) w(x) d x=0 \text { for } j=0, \ldots, n+m
$$


if and only if

$$
\frac{1}{k_{n} E_{n+1}(y)}=q_{n}(y, w)+O\left(y^{-(2 n+3+m)}\right)
$$

for sufficiently large $|y|$.

Proof. Necessity. On the one hand, we have by the orthogonality property of $p_{n}(\cdot, w)$ that

$$
\begin{gathered}
\int_{-1}^{1} \frac{E_{n+1}(y)-E_{n+1}(x)}{y-x} p_{n}(x, w) w(x) d x \\
\quad=\int_{-1}^{1} x^{n} p_{n}(x, w) w(x) d x=\frac{1}{k_{n}},
\end{gathered}
$$

and, on the other hand, setting

$$
E_{n+1}^{(1)}(y)=\int_{-1}^{1} \frac{E_{n+1}(y)-E_{n+1}(x)}{y-x} p_{n}(x, w) w(x) d x
$$

and using the orthogonality property of $E_{n+1}$, we have that

$$
\begin{aligned}
\frac{E_{n+1}^{(1)}(y)}{E_{n+1}(y)}= & q_{n}(y, w)-\int_{-1}^{1} \frac{E_{n+1}(x)}{y-x} p_{n}(x, w) w(x) d x / E_{n+1}(y) \\
= & q_{n}(y, w)-\sum_{j=n+m+1}^{\infty}\left(\int_{-1}^{1} x^{j} E_{n+1}(x) p_{n}(x, w) w(x) d x\right) y^{-(j+1)} \\
& \cdot O\left(y^{-(n+1)}\right) .
\end{aligned}
$$

Sufficiency. Set

$$
k_{n} E_{n+1}(y)=\sum_{j=0}^{n+1} d_{j, n+1} y^{n+1-j},
$$

where $d_{0, n+1}=k_{n}$. Putting $x=1 / y$, it follows from (2.3), with the help of (2.1), that for sufficiently small $|x|$

$$
\frac{1}{\sum_{j=0}^{n+1} d_{j, n+1} x^{j}}=\sum_{j=0}^{\infty}\left(\int_{-1}^{1} t^{j+n} p_{n}(t, w) w(t) d t\right) x^{j}+O\left(x^{n+2+m}\right) .
$$

Equating coefficients and setting

$$
c_{j}=\int_{-1}^{1} t^{j+n} p_{n}(t, w) w(t) d t \quad \text { for } j \in \mathbb{N}_{0}
$$

and

$$
d_{j, n+1}=0 \text { for } j>n+2 \text {, }
$$

we obtain

$$
\sum_{j=0}^{\kappa} d_{j, n+1} c_{\kappa-j}=0 \text { for } \kappa=1,2, \ldots, n+1+m .
$$


Moreover,

$$
\sum_{j=0}^{n+1} d_{j, n+1} c_{\kappa+1-j}=0 \text { for } \kappa=0,1, \ldots, n+m,
$$

which is obviously equivalent to

$$
\int_{-1}^{1} \sum_{j=0}^{n+1} d_{j, n+1} t^{n+1-j} t^{\kappa} p_{n}(t, w) w(t) d t=0 \text { for } \kappa=0,1, \ldots, n+m .
$$

This proves the sufficiency part.

Remark. In view of Lemma 1 , the Stieltjes polynomial $E_{n+1}(\cdot)=E_{n+1}(\cdot, w)$ is given by the (known) relation

$$
k_{n} E_{n+1}(y, w)=\frac{1}{q_{n}(y, w)}+O\left(y^{-1}\right),
$$

for sufficiently large $|y|$.

We recall the following well-known connection between Gauss-Kronrod quadrature formulas and Stieltjes polynomials.

Lemma 2 (see, e.g., [1]). We have

$$
\begin{aligned}
\int_{-1}^{1} s(x) w(x) d x= & \sum_{\nu=1}^{n} \sigma_{\nu, n} s\left(x_{\nu, n}\right) \\
& +\sum_{\mu=1}^{n+1} \lambda_{\mu, n} s\left(y_{\mu, n}\right) \quad \text { for all } s \in \mathbb{P}_{3 n+1}
\end{aligned}
$$

if and only if

$$
\prod_{\mu=1}^{n+1}\left(x-y_{\mu, n}\right)=E_{n+1}(x, w) \text {. }
$$

Furthermore, the Gauss-Kronrod quadrature formula (2.5) is exact for all $s \in$ $\mathbb{P}_{3 n+1+m}, m \in \mathbb{N}_{0}$, if and only if $E_{n+1}(\cdot, w)$ is orthogonal to $\mathbb{P}_{n+m}$ with respect to $p_{n}(\cdot, w) w$ on $[-1,1]$.

The degree of exactness of Gauss-Kronrod quadrature formulas with respect to the Gegenbauer weight function is studied in [16].

\section{ASYMPTOTIC BEHAVIOR OF THE FUNCTIONS OF SECOND KIND}

In order to state our result, we need the following

Notation. Given a polynomial $P_{n}(z)$ of degree $n$, we define the ${ }^{*}$-transform by

$$
P_{n}^{*}(z):=z^{n} \overline{P_{n}(1 / \bar{z})}
$$

so that the coefficient of $z^{j}$ in $P_{n}^{*}$ is the complex conjugate of the coefficient of $z^{n-j}$ in $P_{n}(z), j=0,1,2, \ldots, n$. By

$$
\phi_{n}(z):=\phi_{n}(z, f):=\kappa_{n} z^{n}+\cdots
$$


with $\kappa_{n}:=\kappa_{n}(f), n=0,1,2, \ldots$, we denote the polynomials which are orthogonal on the unit circle $|z|=1$ with respect to the weight function $f \in$ $L_{2}[-\pi, \pi], f \geq 0$ on $[-\pi, \pi]$, i.e.,

$$
\frac{1}{2 \pi} \int_{-\pi}^{\pi} \phi_{n}\left(e^{i \theta}, f\right) \overline{\phi_{m}\left(e^{i \theta}, f\right)} f(\theta) d \theta=\delta_{n, m} \quad \text { for } m, n=0,1,2, \ldots
$$

It is well known that these orthogonal polynomials satisfy a recurrence relation of the form

$$
\kappa_{n}(f) \phi_{n+1}(z, f)=\kappa_{n+1}(f) z \phi_{n}(z, f)+\phi_{n+1}(0, f) \phi_{n}^{*}(z, f),
$$

and the monic orthogonal polynomials

$$
\Phi_{n}(z):=\Phi_{n}(z, f):=\phi_{n}(z) / \kappa_{n}, \quad n=0,1,2, \ldots,
$$

satisfy the recurrence relation

$$
\Phi_{n+1}(z, f)=z \Phi_{n}(z, f)+\Phi_{n+1}(0, f) \Phi_{n}^{*}(z, f) .
$$

The numbers

$$
\begin{aligned}
a_{n} & :=a_{n}(f):=-\Phi_{n+1}(0, f) \\
& =-\phi_{n+1}(0, f) / \kappa_{n+1}(f), \quad n=0,1,2, \ldots,
\end{aligned}
$$

are called reflection coefficients or Schur parameters. It is well known (see [19, (11.3.12) and (11.3.13)]) that

$$
\lim _{n \rightarrow \infty} a_{n}(f)=0
$$

if $\log f(\theta)$ is Lebesgue-integrable on $[-\pi, \pi]$.

In what follows we need the following result of Geronimus ([6, p. 82], resp. [5]), which can be considered as a certain generalization of the so-called SzegöBernstein asymptotic formula for orthonormal polynomials on the unit circle: Assume that the weight function $f$ has a representation of the form

$$
f(\theta)=\left|D\left(e^{i \theta}\right)\right|^{2} \text { for } \theta \in[-\pi, \pi],
$$

where $D(z)$ is analytic and $D(z) \neq 0$ for $|z|<R, R>1$, and $D(0)>0$. Then the following asymptotic formula holds (compare also $[19, \S 12.1]$ ):

$$
\phi_{n}^{*}(z, f)=\frac{1}{D(z)}+\varepsilon_{n}(z)
$$

where

$$
\lim _{n \rightarrow \infty} \varepsilon_{n}(z)=0 \text { uniformly for }|z| \leq r<R .
$$

Furthermore, we have the following connection between polynomials orthonormal on $[-1,1]$ and polynomials $\phi_{n}$ orthonormal on the unit circle (see, e.g., $[19$, p. 294]): Let $w$ be a weight function on $[-1,1]$ and put

$$
f(\theta):=w(\cos \theta)|\sin \theta| \text { for } \theta \in[-\pi, \pi] .
$$


Then the following relations hold for $n \in \mathbb{N}$, where $x=\frac{1}{2}\left(z+z^{-1}\right)$ :

$$
\begin{aligned}
p_{n}(x, w) & =\frac{\left\{z^{-n} \phi_{2 n}(z, f)+z^{n} \phi_{2 n}\left(z^{-1}, f\right)\right\}}{\sqrt{2 \pi\left(1+\phi_{2 n}(0, f) / \kappa_{2 n}(f)\right)}} \\
& =\frac{\left\{z^{-n+1} \phi_{2 n-1}(z, f)+z^{n-1} \phi_{2 n-1}\left(z^{-1}, f\right)\right\}}{\sqrt{2 \pi\left(1-\phi_{2 n}(0, f) / \kappa_{2 n}(f)\right)}}
\end{aligned}
$$

and

$$
\begin{aligned}
p_{n-1}\left(x,\left(1-x^{2}\right) w\right)= & \sqrt{\frac{2}{\pi\left(1-\phi_{2 n}(0, f) / \kappa_{2 n}(f)\right)}} \\
& \cdot\left\{\frac{z^{-n} \phi_{2 n}(z, f)-z^{n} \phi_{2 n}\left(z^{-1}, f\right)}{z-z^{-1}}\right\} \\
= & \sqrt{\frac{2}{\pi\left(1+\phi_{2 n}(0, f) / \kappa_{2 n}(f)\right)}} \\
& \cdot\left\{\frac{z^{-n+1} \phi_{2 n-1}(z, f)-z^{n-1} \phi_{2 n-1}\left(z^{-1}, f\right)}{z-z^{-1}}\right\} .
\end{aligned}
$$

Finally let, as usual, $T_{n}$, resp. $U_{n}$, denote the Chebyshev polynomial of first, resp. second, kind on $[-1,1]$ of degree $n$.

The following theorem plays a crucial role in what follows.

Theorem 1. Suppose that $D$ satisfies the following conditions: $D(z)$ is analytic and $D(z) \neq 0$ for $|z| \leq R$, where $R \geq 1, D$ takes on real values for real $z$, and $D(0)>0$. Furthermore, set

$$
\sin \theta w(\cos \theta):=\left|D\left(e^{i \theta}\right)\right|^{2} \text { for } \theta \in[0, \pi] .
$$

Then for each $n \in \mathbb{N}_{0}, q_{n}\left(\frac{1}{2}\left(z+z^{-1}\right),\left(1-x^{2}\right) w\right) / z^{n+1}$ has an analytic continuation to $|z| \leq R$ and

$$
\lim _{n \rightarrow \infty} \frac{q_{n}\left(\frac{1}{2}\left(z+z^{-1}\right),\left(1-x^{2}\right) w\right)}{z^{n+1}}=\sqrt{2 \pi} D(z) \quad \text { uniformly on }|z| \leq R
$$

Proof. Put

$$
\tilde{p}_{n}(x)=p_{n}\left(x,\left(1-x^{2}\right) w\right)
$$

and

$$
f(\theta)=w(\cos \theta)|\sin \theta| \quad \text { for } \theta \in[-\pi, \pi] .
$$

With the help of the relation

$$
\frac{z}{z^{2}-2 z \cos \theta+1}=\sum_{k=1}^{\infty} \frac{\sin k \theta}{\sin \theta} z^{k} \text { for }|z|<1
$$


we obtain, with $y=\frac{1}{2}\left(z+z^{-1}\right)$,

$$
\begin{aligned}
q_{n}(y, & \left.\left(1-x^{2}\right) w\right)=\int_{-1}^{1} \frac{\tilde{p}_{n}(x)\left(1-x^{2}\right) w(x)}{y-x} d x \\
& =2 z \int_{0}^{\pi} \frac{\tilde{p}_{n}(\cos \theta) \sin ^{2} \theta f(\theta)}{z^{2}-2 z \cos \theta+1} d \theta \\
& =\sum_{k=1}^{\infty}\left(\int_{-\pi}^{\pi} \frac{\sin k \theta}{\sin \theta} \tilde{p}_{n}(\cos \theta) \sin ^{2} \theta f(\theta) d \theta\right) z^{k} \\
& =z^{n+1} \sum_{k=0}^{\infty}\left(\int_{-\pi}^{\pi} \sin (n+k+1) \theta \tilde{p}_{n}(\cos \theta) \sin \theta f(\theta) d \theta\right) z^{k}
\end{aligned}
$$

where the last equality follows from the fact that by the orthogonality property of $\tilde{p}_{n}$,

$$
\int_{-1}^{1} U_{k-1}(x) \tilde{p}_{n}(x)\left(1-x^{2}\right) w(x) d x=0 \text { for } k=1, \ldots, n
$$

Next, we show that for $n \in \mathbb{N}_{0}$ the radius of convergence of the power series

$$
Q_{n}(z):=\sum_{k=0}^{\infty}\left(\int_{-\pi}^{\pi} \sin (n+k+1) \theta \tilde{p}_{n}(\cos \theta) \sin \theta f(\theta) d \theta\right) z^{k}
$$

is greater than, or equal to, $\rho$, where $\rho>R$ is such that $D(z)$ is analytic and $D(z) \neq 0$ for $|z| \leq \rho$. Using the fact [cf. (3.9)] that

$$
2 A_{n} \tilde{p}_{n}(\cos \theta)=\frac{e^{-i(n+1) \theta} \phi_{2 n+2}\left(e^{i \theta}\right)-e^{i(n+1) \theta} \phi_{2 n+2}\left(e^{-i \theta}\right)}{i \sin \theta},
$$

where by (3.2) and (3.3)

$$
2 A_{n}=\sqrt{2 \pi\left(1+a_{2 n+1}\right)} \text { and } \lim _{n \rightarrow \infty} 2 A_{n}=\sqrt{2 \pi},
$$

and noting from (3.5) that

$$
e^{-i(n+1) \theta} \phi_{2 n+2}\left(e^{i \theta}\right)=\frac{e^{i(n+1) \theta}}{D\left(e^{-i \theta}\right)}+e^{i(n+1) \theta} \varepsilon_{2 n+2}\left(e^{-i \theta}\right) \quad \text { for } \theta \in[-\pi, \pi]
$$

where

$$
\lim _{n \rightarrow \infty} \varepsilon_{2 n+2}(z)=0 \quad \text { uniformly on }|z| \leq \tilde{\rho}<\rho,
$$

we get for the $k$ th coefficient of $Q_{n}$ multiplied by $2 A_{n}$, recalling definition 
(3.11), that

$$
\begin{aligned}
2 A_{n} & \int_{-\pi}^{\pi} \sin (n+k+1) \theta \tilde{p}_{n}(\cos \theta) \sin \theta f(\theta) d \theta \\
& =-\frac{1}{2} \int_{-\pi}^{\pi}\left[e^{i(n+k+1) \theta}-e^{-i(n+k+1) \theta}\right] \\
& \cdot\left[e^{-i(n+1) \theta} \phi_{2 n+2}\left(e^{i \theta}\right)-e^{i(n+1) \theta} \phi_{2 n+2}\left(e^{-i \theta}\right)\right] f(\theta) d \theta \\
= & -\int_{-\pi}^{\pi}\left[e^{i(n+k+1) \theta}-e^{-i(n+k+1) \theta}\right] e^{-i(n+1) \theta} \phi_{2 n+2}\left(e^{i \theta}\right) f(\theta) d \theta \\
= & \int_{-\pi}^{\pi} e^{-i k \theta} D\left(e^{i \theta}\right) d \theta+\int_{-\pi}^{\pi} e^{-i k \theta} \varepsilon_{2 n+2}\left(e^{-i \theta}\right) f(\theta) d \theta \\
& -\int_{-\pi}^{\pi} e^{i(2 n+2+k) \theta} \varepsilon_{2 n+2}\left(e^{-i \theta}\right) f(\theta) d \theta,
\end{aligned}
$$

where the last equality follows from the fact that by the analyticity of $D$,

$$
\int_{-\pi}^{\pi} e^{i(2 n+2+k) \theta} D\left(e^{i \theta}\right) d \theta=0 \text { for } n ; k \in \mathbb{N}_{0} .
$$

Now we show that for $\tilde{\rho} \in(0, \rho)$ and $k \in \mathbb{N}_{0}$,

$$
\begin{aligned}
& \mid \int_{-\pi}^{\pi} e^{-i k \theta} \varepsilon_{2 n+2}\left(e^{-i \theta}\right) f(\theta) d \theta \\
& \quad-\int_{-\pi}^{\pi} e^{i(2 n+2+k) \theta} \varepsilon_{2 n+2}\left(e^{-i \theta}\right) f(\theta) d \theta \mid \leq \frac{c M_{2 n+2}(\tilde{\rho})}{\tilde{\rho}^{k}},
\end{aligned}
$$

where $M_{2 n+2}(\tilde{\rho})=\max _{|z| \leq \tilde{\rho}}\left|\varepsilon_{2 n+2}(z)\right|$ and $c$ is a constant not depending on $n$ and $k$. Indeed, since $D$ is analytic and thus, by (3.5), $\varepsilon_{n}, n \in \mathbb{N}_{0}$, is analytic on $|z| \leq \rho$, we have by Cauchy's inequality, setting

$$
D(z)=\sum_{j=0}^{\infty} d_{j} z^{j} \quad \text { and } \quad \varepsilon_{n}(z)=\sum_{j=0}^{\infty} \delta_{j, n} z^{j}
$$

that for $\tilde{\rho} \in(0, \rho]$

$$
\left|d_{j}\right| \leq \max _{|z| \leq \tilde{\rho}}|D(z)| / \tilde{\rho}^{j} \quad \text { and } \quad\left|\delta_{j, n}\right| \leq M_{n}(\tilde{\rho}) / \tilde{\rho}^{j} .
$$

Letting

$$
\left|D\left(e^{i \theta}\right)\right|^{2}=\sum_{j=0}^{\infty} b_{j} \cos j \theta
$$

the first relation of $(3.18)$ implies that for $\tilde{\rho} \in(0, \rho]$

$$
\left|b_{j}\right| \leq c_{1} / \tilde{\rho}^{j} \text { for } j \in \mathbb{N}_{0} .
$$

Similarly, letting $B(z)=\sum_{j=0}^{\infty} b_{j} z^{j}$ (note that by (3.19) $B$ is analytic on $|z| \leq$ $\tilde{\rho}$ for $0<\tilde{\rho}<\rho)$, and using the fact that

$$
\int_{-\pi}^{+\pi} e^{-i k \theta}\left|D\left(e^{i \theta}\right)\right|^{2} \varepsilon_{2 n+2}\left(e^{-i \theta}\right) d \theta=\frac{1}{2} \int_{-\pi}^{\pi} e^{-i k \theta} B\left(e^{i \theta}\right) \varepsilon_{2 n+2}\left(e^{-i \theta}\right) d \theta
$$


we obtain with the help of (3.19) and the second relation of (3.18) that for $\tilde{\rho} \in(0, \rho]$

$$
\left|\int_{-\pi}^{\pi} e^{-i k \theta} \varepsilon_{2 n+2}\left(e^{-i \theta}\right) f(\theta) d \theta\right| \leq \frac{c_{2} M_{2 n+2}(\tilde{\rho})}{\tilde{\rho}^{k}} \text { for } k \in \mathbb{N}_{0},
$$

where $c_{2} \in \mathbb{R}^{+}$. Since the second integral in (3.17) can be split into

$$
\begin{aligned}
& \frac{1}{2} \int_{-\pi}^{\pi} e^{i(2 n+2+k) \theta} B\left(e^{i \theta}\right) \varepsilon_{2 n+2}\left(e^{-i \theta}\right) d \theta \\
& \quad+\frac{1}{2} \int_{-\pi}^{\pi} e^{i(2 n+2+k) \theta} B\left(e^{-i \theta}\right) \varepsilon_{2 n+2}\left(e^{-i \theta}\right) d \theta,
\end{aligned}
$$

it follows as above that the absolute value of the first integral is smaller than $c_{3} M_{2 n+2}(\tilde{\rho}) / \tilde{\rho}^{2 n+2+k}$ for $\tilde{\rho} \in(0, \rho]$ and finally by the analyticity of $B \varepsilon_{2 n+2}$ on $|z| \leq \tilde{\rho}, \tilde{\rho}<\rho$, that the absolute value of the second integral is smaller than $c_{4} M_{2 n+2}(\tilde{\rho}) / \tilde{\rho}^{2 n+2+k}$ for $\tilde{\rho} \in(0, \rho)$. Thus, relation (3.17) is proved. This, in conjunction with (3.16) and the fact that $D$ is analytic on $|z| \leq \rho$, implies that the radius of convergence of $Q_{n}$ is greater than $R$, which proves the first statement of the theorem.

Concerning the uniform convergence, we use

$$
D(z)=\sum_{k=0}^{\infty}\left(\frac{1}{2 \pi} \int_{-\pi}^{\pi} e^{-i k \varphi} D\left(e^{i \varphi}\right) d \varphi\right) z^{k}
$$

together with (3.13), (3.16), and (3.17) to show that $R<\tilde{\rho}<\rho$ and

$$
\left|\frac{A_{n}}{\pi} Q_{n}(z)-D(z)\right| \leq c M_{2 n+2}(\tilde{\rho}) \sum_{k=0}^{\infty}\left(\frac{|z|}{\tilde{\rho}}\right)^{k} .
$$

In view of (3.15) and the second relation of (3.14), this yields the assertion.

Remark. Geronimus pointed out in [7, p. 102] that, under the assumption that the weight function satisfies Szegö's condition, one gets with the help of the relation

$$
\lim _{n \rightarrow \infty} p_{n}(x, w) q_{n}(x, w)=\frac{1}{\sqrt{x^{2}-1}} \text { for } x \notin[-1,1]
$$

and the asymptotic formula of Szegö-Bernstein for $p_{n}(x, w)$ that, with $x=$ $\frac{1}{2}\left(z+z^{-1}\right)$

$$
\frac{q_{n}(x, w)}{z^{n}} \cong \frac{\sqrt{2 \pi} D(z)}{z^{-1}-z} \text { for }|z| \leq 1-\varepsilon, \varepsilon>0 .
$$

But note that relation (3.22) gives an asymptotic representation in the interior of the unit circle only, while for our purpose we need an asymptotic representation which is valid also outside the unit circle. Unfortunately, it seems to be impossible to get Theorem 1 in a similar way as relation (3.22). 


\section{Zeros of Stieltues polynomials}

In this section we prove the main result of this paper, namely that under suitable conditions on the weight function the Stieltjes polynomial $E_{n}(\cdot, w)$ has $n$ simple zeros in $(-1,1)$.

Lemma 3. Let $E_{n+1}(x)=x^{n+1}+\cdots$ and $G_{n+1}(z)=z^{n+1}+\cdots$ be real polynomials of degree $n+1$ such that

$$
E_{n+1}(x)=2^{-n} \operatorname{Re}\left\{G_{n+1}\left(e^{i \theta}\right)\right\}, \quad \text { where } x=\cos \theta, \theta \in[0, \pi] .
$$

Then

$$
\int_{-1}^{1} x^{j} E_{n+1}(x) p_{n}(x, w) w(x) d x=0 \text { for } j=0,1, \ldots, n+m
$$

if and only if

$$
z^{n+1} G_{n+1}(z)+G_{n+1}^{*}(z)=\frac{2^{n+1}}{k_{n}} \frac{z^{n+1}}{q_{n}\left(\frac{1}{2}\left(z+z^{-1}\right), w\right)}+O\left(z^{n+2+m}\right)
$$

for sufficiently small $|z|, z \in \mathbb{C}$.

Proof. Let

$$
G_{n+1}(z)=\sum_{j=0}^{n+1} d_{j} z^{n+1-j} .
$$

Then we get from (4.1) that

$$
2^{n} E_{n+1}(y)=\sum_{j=0}^{n+1} d_{j} T_{n+1-j}(y) .
$$

Setting $z=y-\sqrt{y^{2}-1}$ for $y \in \mathbb{C} \backslash[-1,1]$, i.e., $y=\frac{1}{2}\left(z+z^{-1}\right)$ with $|z|<1$, and recalling

$$
T_{n}(y)=\frac{\left(y+\sqrt{y^{2}-1}\right)^{n}+\left(y-\sqrt{y^{2}-1}\right)^{n}}{2},
$$

we obtain that

$$
(2 z)^{n+1} E_{n+1}(y)=z^{n+1} G_{n+1}(z)+G_{n+1}^{*}(z)
$$

and thus, by Lemma 1 ,

$$
\frac{1}{(2 z)^{n+1} E_{n+1}(y)}=\frac{k_{n}}{2^{n+1}} \frac{q_{n}\left(\frac{1}{2}\left(z+z^{-1}\right), w\right)}{z^{n+1}}+O\left(z^{n+2+m}\right),
$$

which gives the assertion. 
The main result is now the following

Theorem 2. Suppose that $D(z)$ is analytic and $D(z) \neq 0$ for $|z| \leq 1$, and that $D$ takes on real values for real $z$. Furthermore, set

$$
w(\cos \theta) \sin \theta:=\left|D\left(e^{i \theta}\right)\right|^{2} \quad \text { for } \theta \in(0, \pi) .
$$

Then there exists an $n_{0} \in \mathbb{N}$ such that for each $n \geq n_{0}$ the Stieltjes polynomial $E_{n}\left(\cdot,\left(1-x^{2}\right) w\right)$ has $n$ simple zeros in $(-1,1)$.

Proof. Let us assume, without loss of generality, that $D(0)>0$, and put

$$
\widetilde{Q}_{n}(z):=\frac{2^{n+1}}{k_{n}} \frac{z^{n+1}}{q_{n}\left(\frac{1}{2}\left(z+z^{-1}\right),\left(1-x^{2}\right) w\right)} .
$$

Since $G_{n+1}^{*}(0)=1$, we get from relation (4.2) and Theorem 1 that

$$
\lim _{n \rightarrow \infty} \frac{2^{n+1}}{k_{n}} \frac{1}{\sqrt{2 \pi} D(0)}=1
$$

By Theorem 1 this yields

$$
\lim _{n \rightarrow \infty} \widetilde{Q}_{n}(z)=\frac{D(0)}{D(z)}=: \frac{1}{\widehat{D}(z)} \quad \text { uniformly on }|z| \leq 1 .
$$

We thus obtain from (4.2) and (4.3) by standard arguments of uniform convergence, using also the fact that, by $(4.2), G_{n+1}^{*}(z)+G_{n+1}(0) z^{n+1}$ is the $(n+1)$ st partial sum of $\widetilde{Q}_{n}(z)$, that for given $\varepsilon_{1}>0$ there is an $n_{1} \in \mathbb{N}$ such that for $n \geq n_{1}$ and $|z| \leq 1$

$$
\left|G_{n+1}^{*}(z)+G_{n+1}(0) z^{n+1}-1 / \widehat{D}(z)\right|<\varepsilon_{1} .
$$

Applying Cauchy's integral formula on $|z|=1$ to the $(n+1)$ st derivative of the analytic functions $\widetilde{Q}_{n}$ and $1 / \widehat{D}$ at the point zero, we get with the help of (4.3) that for any $\varepsilon_{2}>0$ there is an $n_{2}$ such that $n \geq n_{2}$ implies

$$
\left|2 G_{n+1}(0)-\hat{d}_{n+1}\right|<\varepsilon_{2},
$$

where $\hat{d}_{n+1}=(1 / \widehat{D})^{(n+1)}(0) /(n+1)$ !. Now, since $1 / \widehat{D}$ is analytic on $|z| \leq 1$, we have $\lim _{n \rightarrow \infty} \hat{d}_{n+1}=0$ and thus by (4.5) that

$$
\lim _{n \rightarrow \infty} G_{n+1}(0)=0 \text {. }
$$

Hence, in view of (4.4), we find that for given $\varepsilon>0$ there exists an $n_{0}$ such that for $n \geq n_{0}$ and $|z| \leq 1$

$$
\left|G_{n+1}^{*}(z)-1 / \widehat{D}(z)\right|<\varepsilon .
$$

Recalling $\widehat{D}(z) \neq 0$ for $|z| \leq 1,(4.6)$ immediately implies that $G_{n+1}^{*}(z)$ for large $n$ has no zero in $|z| \leq 1$, which is equivalent to the fact that $G_{n+1}$ has all zeros in $|z|<1$. Hence (see, e.g., [15, Lemma 2]), $E_{n+1}\left(x,\left(1-x^{2}\right) w\right)=$ $2^{-n} \operatorname{Re}\left\{G_{n+1}\left(e^{i \theta}\right)\right\}, x=\cos \theta$, has $n+1$ simple zeros in $(-1,1)$, which proves the theorem. 
In view of Szegö's results [18] mentioned in the introduction, it is likely that Theorem 2 holds true for weight functions of the type

$$
w(x)=\left(1-x^{2}\right)^{\lambda-3 / 2}\left|D\left(e^{i \theta}\right)\right|^{2}, \quad 0<\lambda \leq 2,
$$

$x=\cos \theta, \theta \in(0, \pi)$, where $D$ satisfies the assumptions of Theorem 2 .

The following asymptotic representation of the Stieltjes polynomial is important in what follows.

Corollary 1. Let $k_{n+1}^{\prime}$ be the leading coefficient of $p_{n+1}(x, w)$ and suppose that the assumptions of Theorem 2 are fulfilled. Then the Stieltjes polynomial has the following asymptotic representation on $[-1,1]$ :

$$
k_{n+1}^{\prime} E_{n+1}\left(x,\left(1-x^{2}\right) w\right)=p_{n+1}(x, w)+\delta_{n+1}(x) \text { for } x \in[-1,1],
$$

where $\lim _{n \rightarrow \infty} \delta_{n}(x)=0$ uniformly on $[-1,1]$.

Proof. In view of relation (4.6) we have

$$
G_{n+1}^{*}\left(e^{i \theta}\right)=\frac{1}{\widehat{D}\left(e^{i \theta}\right)}+\eta_{n+1}\left(e^{i \theta}\right),
$$

where $\lim _{n \rightarrow \infty} \eta_{n+1}\left(e^{i \theta}\right)=0$ uniformly on $[-\pi, \pi]$ and $\hat{D}$ is defined as in (4.3). This implies

$$
G_{n+1}\left(e^{i \theta}\right)=\frac{e^{i(n+1) \theta}}{\widehat{D}\left(e^{-i \theta}\right)}+\tilde{\eta}_{n+1}\left(e^{i \theta}\right)
$$

where $\lim _{n \rightarrow \infty} \tilde{\eta}_{n+1}\left(e^{i \theta}\right)=0$ uniformly on $[-\pi, \pi]$. Recalling that by $(3.5)$ and (3.6)

$$
\Phi_{2 n+1}\left(e^{i \theta},\left|D\left(e^{i \theta}\right)\right|^{2}\right)=\frac{e^{i(2 n+1) \theta}}{\widehat{D}\left(e^{-i \theta}\right)}+\tilde{\varepsilon}_{2 n+1}\left(e^{i \theta}\right),
$$

where $\lim _{n \rightarrow \infty} \tilde{\varepsilon}_{2 n+1}\left(e^{i \theta}\right)=0$ uniformly on $[-\pi, \pi]$, we get with the help of (3.8) that

$$
\begin{aligned}
2^{n+1} & E_{n+1}\left(x,\left(1-x^{2}\right) w\right)=2 \operatorname{Re}\left\{G_{n+1}\left(e^{i \theta}\right)\right\} \\
& =2 \operatorname{Re}\left\{\frac{e^{i(n+1) \theta}}{\widehat{D}\left(e^{-i \theta}\right)}\right\}+\gamma_{n+1}(\cos \theta) \\
& =2 \operatorname{Re}\left\{e^{-i n \theta} \Phi_{2 n+1}\left(e^{i \theta},\left|D\left(e^{i \theta}\right)\right|^{2}\right)\right\}+\tilde{\delta}_{n+1}(\cos \theta) \\
& =2^{n+1} P_{n+1}(x, w)+\tilde{\delta}_{n+1}(\cos \theta),
\end{aligned}
$$

where $\lim _{n \rightarrow \infty} \gamma_{n+1}(\cos \theta)=0$ and $\lim _{n \rightarrow \infty} \tilde{\delta}_{n+1}(\cos \theta)=0$ uniformly on $[0, \pi]$. Using the fact that by [19, Theorem 2.7.1], $k_{n+1}^{\prime}=O\left(2^{n+1}\right)$, since $\left|D\left(e^{i \theta}\right)\right|^{2}$ is in the Szegö class, the corollary follows.

If the weight function $w$ is of the form $w(x)=1 /\left(1-x^{2}\right)^{1 / 2} s_{m}(x)$, where $s_{m}$ is a positive polynomial on $[-1,1]$ of degree $m$, we even have equality in Corollary 1 for $n \geq m$ (see [13 or 14]). 
The second main result of this paper is the following

Theorem 3. Suppose that the assumptions of Theorem 2 are fulfilled. Then there exists an $n_{0} \in \mathbb{N}$ such that the following propositions hold for each $n \geq n_{0}$ :

(a) The zeros of $E_{n+1}\left(\cdot,\left(1-x^{2}\right) w\right)$ and $p_{n}\left(\cdot,\left(1-x^{2}\right) w\right)$ separate each other.

(b) All quadrature weights $\sigma_{\nu, n}, \nu=1, \ldots, n$, and $\lambda_{\mu, n}, \mu=1, \ldots, n+$ 1 , of the Gauss-Kronrod quadrature formula (1.2) are positive.

Proof. (a) Using (4.7) and (4.8) together with the fact that $1 /\left|D\left(e^{i \theta}\right)\right|$ is bounded on $[-\pi, \pi]$, we obtain (note that the first equality follows by simple calculation)

$$
\begin{aligned}
\operatorname{Re}\left\{G_{n+1}\right. & \left.\left(e^{i \theta}\right)\right\} \operatorname{Re}\left\{e^{-i(n+1) \theta} \Phi_{2 n+2}\left(e^{i \theta}\right)\right\} \\
& +\operatorname{Im}\left\{G_{n+1}\left(e^{i \theta}\right)\right\} \operatorname{Im}\left\{e^{-i(n+1) \theta} \Phi_{2 n+2}\left(e^{i \theta}\right)\right\} \\
= & \operatorname{Re}\left\{G_{n+1}\left(e^{i \theta}\right) e^{i(n+1) \theta} \Phi_{2 n+2}\left(e^{-i \theta}\right)\right\} \\
& -\frac{1}{\left|\widehat{D}\left(e^{i \theta}\right)\right|^{2}}+\delta_{n+1}\left(e^{i \theta}\right)>0 \text { for } n \geq n_{0},
\end{aligned}
$$

where $\lim _{n \rightarrow \infty} \delta_{n+1}\left(e^{i \theta}\right)=0$ uniformly on $[-\pi, \pi]$. Observing that by [15, Lemma 2] the zeros of $\operatorname{Re}\left\{G_{n+1}\left(e^{i \theta}\right)\right\}$ and $\operatorname{Im}\left\{G_{n+1}\left(e^{i \theta}\right)\right\} / \sin \theta$ separate each other, since $G_{n+1}$ has all zeros in the open unit disk $|z|<1$, we get, using relation (4.9) at the zeros of $\operatorname{Re}\left\{G_{n+1}\left(e^{i \theta}\right)\right\}$, that the zeros of $\operatorname{Re}\left\{G_{n+1}\left(e^{i \theta}\right)\right\}$ and $\operatorname{Im}\left\{e^{-i(n+1) \theta} \Phi_{2 n+2}\left(e^{i \theta}\right)\right\} / \sin \theta$ separate each other. In view of (3.9), this proves part (a).

(b) By [10, Theorem 1], (a) is equivalent to the positivity of the $\lambda_{\mu, n}$ 's. Thus, it remains to show that (compare [10, Theorem 2 and 4, (6.4)])

$$
\begin{aligned}
\sigma_{\nu, n} & =c_{\nu, n}+\frac{1}{\tilde{k}_{n}^{2} E_{n+1}\left(x_{\nu, n}\right) \widetilde{P}_{n}^{\prime}\left(x_{\nu, n}\right)} \\
& =\frac{-1}{\tilde{k}_{n}^{2} E_{n+1}\left(x_{\nu, n}\right) \widetilde{P}_{n}^{\prime}\left(x_{\nu, n}\right)} \cdot\left(\frac{E_{n+1}\left(x_{\nu, n}\right)}{\widetilde{P}_{n+1}\left(x_{\nu, n}\right)}-1\right)>0
\end{aligned}
$$

for $\nu=1, \ldots, n$, where $c_{\nu, n}$ denotes the weights of the $n$-point Gauss quadrature formula relative to the weight function $\left(1-x^{2}\right) w, P_{n}(\cdot):=P_{n}(\cdot, w)$, resp. $\widetilde{P}_{n}(\cdot):=P_{n}\left(\cdot,\left(1-x^{2}\right) w\right)$, is the monic orthogonal polynomial with respect to $w$, resp. $\left(1-x^{2}\right) w, \tilde{k}_{n}^{2}=\int_{-1}^{1} \widetilde{P}_{n}^{2}\left(1-x^{2}\right) w d x$, and $x_{\nu, n}$ is the $\nu$ th zero of $\widetilde{P}_{n}$. Because of part (a), the positivity of $\sigma_{\nu, n}$ is equivalent to the inequality

$$
\frac{E_{n+1}\left(x_{\nu, n}\right)}{\widetilde{P}_{n+1}\left(x_{\nu, n}\right)}>1 \text {. }
$$


In order to prove relation (4.10) for large $n$, we first observe that the following relation holds for $n \in \mathbb{N}$ :

$$
\frac{P_{n+1}\left(x_{\nu, n}\right)}{\widetilde{P}_{n+1}\left(x_{\nu, n}\right)}=\frac{2}{1-a_{2 n+1}},
$$

where $a_{2 n+1}$ is the $(2 n+1)$ st reflection coefficient relative to the weight function $\left|D\left(e^{i \theta}\right)\right|^{2}$. In fact, from the representation

$$
2^{n} \widetilde{P}_{n}(x)=\frac{z^{-(n+1)}\left(z \Phi_{2 n+1}(z)-\Phi_{2 n+1}^{*}(z)\right)}{2 i \sin \theta}
$$

it follows that at the zeros $x_{\nu, n}=\cos \theta_{\nu, n}$ the relation

$$
z_{\nu, n} \Phi_{2 n+1}\left(z_{\nu, n}\right)=\Phi_{2 n+1}^{*}\left(z_{\nu, n}\right)
$$

holds, where $z_{\nu, n}=e^{i \theta_{\nu, n}}, \nu=1, \ldots, n$. Applying the recurrence formula (3.1), resp. the equivalent recurrence formula

$$
\Phi_{n+1}^{*}(z)=\Phi_{n}^{*}(z)-a_{n} z \Phi_{n}(z),
$$

to $\Phi_{2 n+3}$, resp. $\Phi_{2 n+3}^{*}$, and then to $\Phi_{2 n+2}$, resp. $\Phi_{2 n+2}^{*}$, we obtain with the help of (4.13) that

$$
z_{\nu, n} \Phi_{2 n+3}\left(z_{\nu, n}\right)-\Phi_{2 n+3}^{*}\left(z_{\nu, n}\right)=\left(1-a_{2 n+1}\right)\left(z_{\nu, n}^{2}-1\right) \Phi_{2 n+1}^{*}\left(z_{\nu, n}\right)
$$

and thus by (4.12)

$$
2^{n+1} \widetilde{P}_{n+1}\left(x_{\nu, n}\right)=\left(1-a_{2 n+1}\right) z_{\nu, n}^{-(n+1)} \Phi_{2 n+1}^{*}\left(z_{\nu, n}\right) .
$$

This, in conjunction with the relation

$$
\begin{aligned}
2^{n} P_{n+1}\left(x_{\nu, n}\right) & =z_{\nu, n}^{-(n+1)}\left(z \Phi_{2 n+1}+\Phi_{2 n+1}^{*}\right)\left(z_{\nu, n}\right) / 2 \\
& =z_{\nu, n}^{-(n+1)} \Phi_{2 n+1}^{*}\left(z_{\nu, n}\right),
\end{aligned}
$$

proves relation (4.11).

Now taking into account the fact that by (3.6)

$$
\lim _{n \rightarrow \infty}\left|\Phi_{n}^{*}\left(e^{i \theta}\right)\right|=\frac{1}{\left|\widehat{D}\left(e^{i \theta}\right)\right|} \quad \text { uniformly on }[-\pi, \pi],
$$

it follows that $\left|\Phi_{n}^{*}\left(e^{i \theta}\right)\right|$ is bounded from below for sufficiently large $n$, which implies by (4.14) that also $\left|2^{n+1} \widetilde{P}_{n+1}\left(x_{\nu, n}\right)\right|$ is bounded from below for sufficiently large $n$. Since by Corollary $1,2^{n} E_{n+1}(x)=2^{n} P_{n+1}(x)+\delta_{n+1}(x)$, $x \in[-1,1]$, it follows from (4.11) together with (3.3) that relation (4.10) holds for $n \geq n_{0}$. This proves the theorem.

\section{BIBLIOGRAPHY}

1. W. Gautschi, Gauss-Kronrod quadrature-a survey, Numerical Methods and Approximation Theory III (G. V. Milovanović, ed.), University of Niš, Niš, 1988, pp. 39-66.

2. W. Gautschi and S. E. Notaris, An algebraic study of Gauss-Kronrod quadrature formulae for Jacobi weight functions, Math. Comp. 51 (1988), 231-248. 
3. W. Gautschi and T. J. Rivlin, A family of Gauss-Kronrod quadrature formulae, Math. Comp. 51 (1988), 749-754.

4. W. Gautschi and S. E. Notaris, Gauss-Kronrod quadrature formulae for weight functions of Bernstein-Szegö type, J. Comput. Appl. Math. 25 (1989), 199-224. [Erratum: ibid. 27 (1989), 429.]

5. Ya. L. Geronimus, On asymptotic formulae for orthogonal polynomials, Izv. Akad. Nauk SSSR Ser. Mat. 12 (1948), 3-14. (Russian)

6. __ On asymptotic properties of polynomials which are orthogonal on the unit circle, and on certain properties of positive harmonic functions, Izv. Akad. Nauk SSSR Ser. Mat. 14 (1950), 123-144; English transl., Amer. Math. Soc. Transl. (1) 3 (1962), 79-106.

7. __ Orthogonal polynomials, Amer. Math. Soc. Transl. (2) 108 (1977), 37-130.

8. A. S. Kronrod, Nodes and weights for quadrature formulae. Sixteen-place Tables, "Nauka", Moscow, 1964; English transl., Consultants Bureau, New York, 1965.

9. M. Marden, Geometry of polynomials, Math. Surveys, vol. 3, Amer. Math. Soc., Providence, R.I., 1966.

10. G. Monegato, A note on extended Gaussian quadrature rules, Math. Comp. 30 (1976), $812-$ 817.

11. _ Positivity of the weights of extended Gauss-Legendre quadrature rules, Math. Comp. 32 (1978), 243-245.

12. __ Stieltjes polynomials and related quadrature rules, SIAM Rev. 24 (1982), 137-158.

13. S. E. Notaris, Gauss-Kronrod quadrature formulae for weight functions of Bernstein-Szegö type. II, J. Comput. Appl. Math. 29 (1990), 161-170.

14. F. Peherstorfer, Weight functions admitting repeated positive Kronrod quadrature, BIT 30 (1990), 145-151.

15. L Linear combinations of orthogonal polynomials generating positive quadrature formulas, Math. Comp. 55 (1990), 231-241.

16. P. Rabinowitz, The exact degree of precision of generalized Gauss-Kronrod integration rules, Math. Comp. 35 (1980), 1275-1283. [Corrigendum: ibid. 46 (1986), 226, footnote.]

17. __ Gauss-Kronrod integration rules for Cauchy principal value integrals, Math. Comp. 41 (1983), 63-78. [Corrigenda: ibid. 45 (1985), 277.]

18. G. Szegö, Über gewisse orthogonale Polynome, die zu einer oszillierenden Belegungsfunktion gehören, Math. Ann. 110 (1935), 501-513. (Also appears in his Collected papers (R. Askey, ed.), vol. 2, Birkhäuser, Boston-Basel-Stuttgart, 1982, pp. 545-557.)

19. __ Orthogonal polynomials, 3rd ed., Amer. Math. Soc. Colloq. Publ., vol. 23, Amer. Math. Soc., Providence, R.I., 1967.

InSTITUt FÜr MATHEMATIK, J. KePLER UNiversität Linz, A-4040 LiNZ, AUSTRIA 